\title{
Inflammatory Cell Markers in Local Allergic Rhinitis
}

\author{
Şenol Çomoğlu' ${ }^{1 *}$ Ece Çomoğlü, M.Nesil Keleş ${ }^{1}$ and Kemal Değer ${ }^{1}$ \\ ${ }^{1}$ Otorhinolaryngology Head \& Neck Surgery Department Istanbul University, Faculty of Medicine, Istanbul, Turkey
}

${ }^{2}$ Kağıthane State Hospital, İstanbul, Turkey

*Corresponding author: Şenol Çomoğlu, Faculty of Medicine, Department of Otorhinolaryngology Head and Neck Surgery, İstanbul University, Istanbul-Turkey, Tel: +902124142000 (31772), E-mail: drcomoglu@gmail.com,senolcomoglu@outlook.com,senol.comoglu@istanbul.edu.tr

\begin{abstract}
Background: Aim of this study is to detect inflammatory characteristics of idiopathic rhinitis.

Methodology/Principal: The inflammatory cells of the nasal mucosa in patients with idiopathic rhinitis $(n=17)$ were compared with Th2-type inflammatory cells in patients with allergic rhinitis $(n=16)$. Control patients were selected among those who had undergone surgery due to nasal septum deviation and had no complaints of rhinitis $(n=25)$. The number of leukocyte-lymphocyte marker-positive cells per $\mathrm{mm}^{2}$ was counted in the inferior nasal concha specimens. CD1+, CD3+, CD4+, CD8+, CD14+, CD25+ and CD68+ cells were compared respectively. All cells are counted in three layer of nasal mucosa; epithelium, superficial submucosa and deep submucosa.
\end{abstract}

Results: Patients with idiopathic rhinitis have similar inflammatory CD1+, CD3+, CD4+, CD8+, CD14+ and CD25+ cell accumulations in distinct part of nasal mucosa. CD1+ (mean $54,76 \mathrm{cell} / \mathrm{mm}^{2}$ in idiopathic and 51,19 in allergic rhinitis), CD3+ (mean 576,41 cell/ $\mathrm{mm}^{2}$ in idiopathic and 542,94 in allergic rhinitis), CD4+ (mean $358,76 \mathrm{cell} / \mathrm{mm}^{2}$ in idiopathic and 295,56 in allergic rhinitis), CD14+ (mean 193,94 cell/ $\mathrm{mm}^{2}$ in idiopathic and 222.31 in allergic rhinitis), CD25+ (mean $0,76 \mathrm{cell} / \mathrm{mm}^{2}$ in idiopathic and 0,31 in allergic rhinitis) and CD68+ (mean $124,71 \mathrm{cell} / \mathrm{mm}^{2}$ in idiopathic and 116,81 in allergic rhinitis) cells were similarly distributed in epithelial, CD3+ (mean 529,53 cell/ $/ \mathrm{mm}^{2}$ in idiopathic and 478,38 in allergic rhinitis), CD4+ (mean $304,76 \mathrm{cell} / \mathrm{mm}^{2}$ in idiopathic and 279,63 in allergic rhinitis), CD14+ (mean $175,29 \mathrm{cell} / \mathrm{mm}^{2}$ in idiopathic and 183,25 in allergic rhinitis), CD25+ (mean 1,59 cell/ $\mathrm{mm}^{2}$ in idiopathic and 1,75 in allergic rhinitis) and CD68+ (mean $104,76 \mathrm{cell} / \mathrm{mm}^{2}$ in idiopathic and 103,13 in allergic rhinitis) cells were similarly distributed in superficial subepithelial and CD1+ (mean $5,82 \mathrm{cell} / \mathrm{mm}^{2}$ in idiopathic and 4,19 in allergic rhinitis), CD3+ (mean $482,76 \mathrm{cell} / \mathrm{mm}^{2}$ in idiopathic and 446,69 in allergic rhinitis), CD14+ (mean 160,18 cell/mm ${ }^{2}$ in idiopathic and 159,25 in allergic rhinitis), CD25+ (mean $0,88 \mathrm{cell} / \mathrm{mm}^{2}$ in idiopathic and 1,88 in allergic rhinitis) and CD68+ (mean $92,71 \mathrm{cell} / \mathrm{mm}^{2}$ in idiopathic and 83,19 in allergic rhinitis) cells were similarly distributed in deep submucosa with compared to allergic counterparts.

Conclusion: A positive inflammatory reaction, suggesting a localized allergy was observed in patients with idiopathic rhinitis. The reason for the lack of systemic response in patients with idiopathic rhinitis still remains unknown.

\section{Keywords}

Rhinitis, Vasomotor rhinitis, Inflammation, Immune markers, Hypersensitivity

\section{Introduction}

Idiopathic rhinitis (IR), previously referred to as "intrinsic rhinitis" or "non-allergic perennial rhinitis", is a form of rhinitis that occurs without a known predisposing factor [1]. In fact, IR is a subtype of non-allergic non-infectious rhinitis; the pathophysiology of IR has not been completely elucidated. Negative skin-prick test (SPT) along with the classical symptoms at the time of presentation may lead to misdiagnosis of vasomotor rhinitis. However, vasomotor rhinitis is a form of non-allergic rhinitis, which is commonly encountered among the elderly population ( $>60$ years of age) and primarily manifest with clear rhinorrhea rather than nasal obstruction and itching. Patients with IR are diagnosed by excluding other forms of non-allergic rhinitis. Patients with IR have no evidence of atopy, indicated by negative results in SPT and serum specific IgE.

Recently, many studies have been conducted to elucidate the mechanisms that cause symptoms in patients with IR, and these studies have emphasized a potential local allergy in the nasal mucosa, which cannot be determined by SPT [2-5]. It has recently been suggested that IgE is locally synthesized in the nasal mucosa [5] and may cause mucosal changes independent of serum-specific IgE [6]. Moreover, various studies have reported that a localized allergic reaction which does not cause a systemic response is responsible for the pathophysiology of IR. Powe et al. [7] studied antigen-binding antibodies specific for house dust mite and grass pollen allergen in nasal mucosal biopsy specimens and reported a localized allergic response, which was coined "entopy." Carney et al. [2] demonstrated a positive reaction following nasal provocation with house dust mite allergen, suggesting a localized allergic response in patients with IR. In a recent study, findings indicating local production of $\operatorname{IgE}$ and mast cell/eosinophil activation following nasal allergen provocation in patients with IR were obtained [8]. In another recent study, the increase in free light chain immunoglobulin in nasal secretions of subjects with non-atopic rhinitis suggested a role of free light chain 
in nasal hypersensitivity [9]. In this study we aimed to find out the qualification of cellular infiltrates detected in inferior turbinate specimens of patients who are previously diagnosed as 'idipoathic rhinitis' and compare them with 'allergic' counterparts and 'control' subjects as well.

\section{Materials and Methods}

\section{Patient Selection}

Patients, admitted with symptoms of rhinitis, such as rhinorrhea, nasal obstruction, and sneezing, with a symptom duration $>2$ years were included in the study. The patients with infectious etiology were excluded. SPT was performed. Topical anesthesia was applied to the tip of the inferior nasal concha (a piece of cotton absorbed with 20$30 \mathrm{mg}$ of $10 \%$ lidocaine and 2 drops of 1:1000 adrenaline was placed in the inferio-medial aspect of the inferior nasal concha for 5-10 minutes) and biopsy was obtained by using Gerritsma forceps with a cup diameter of $2.5 \mathrm{~mm}$.

Control patients were selected among those who had undergone surgery due to nasal septum deviation and had no complaints or physical findings of rhinitis. Mucosal tissue samples were obtained under general anesthesia with Gerritsma forceps after topical lidocaine application, as previously described.

SPT was performed on the volar surface of the forearm by subcutaneous application of the negative and positive control solutions, together with the 13 most frequently encountered specific aeroallergens (house dust mites, grass pollens, tree pollens, fungi, sweet woodruff, and cat, dog, and bird feather antigens). All of the participants underwent SPT.

Patients with allergic rhinitis (AR) were defined as those with positive SPT without seasonal complaints, whereas the patients with IR were defined as those with negative SPT despite persistent symptoms. Control patients had no symptoms and they had negative results in SPT.

\section{The inclusion criteria for patients with IR were as follows}

$\checkmark 16-60$ years of age,

$\checkmark$ negative SPT to aeroallergens,

$\checkmark$ presence of at least 2 from 3 symptoms of rhinitis:

$\rightarrow$ neither purulent discharge nor intranasal pathology on endoscopic examination,

$\rightarrow$ rhinorrhea, nasal obstruction, and sneezing for at least one hour per day for 14 consecutive days, and

$\rightarrow$ no history of upper respiratory tract infection within the last 1 month, and

$\checkmark$ Presence of symptoms for $>2$ years.

\section{The exclusion criteria for the patients with IR were as follows}

- positive SPT,

- presence of nasal polyps,

- significant anatomic deformity effecting nasal functions,

- presence of paranasal infections,

- pregnancy or lactation,

- continuous use of the drugs effecting nasal functions,

- smoking within the last 6 months,

- use of nasal or systemic corticosteroids within the last 6 weeks

- use of nedocromil sodium or sodium cromoglycate within the last 1 month, and

\section{Tissue processing and immunohistochemical staining}

Biopsy specimens were fixed in $10 \%$ formalin, and routinely processed for paraffin embedding. Mucosal samples were orientated to obtain transverse sections of the epithelium and full-thickness submucosa. Serial $4 \mu \mathrm{m}$ thick sections were prepared. In order to avoid spilling, sections were mounted on poly-L-lysine-coated slides. One slide was stained with hematoxylin, and the others were reserved for immunohistochemical staining. The immunohistochemical examination was performed with microwave heating in combination with UltraTek HRP Anti-Polyvalent Staining System (ScyTec Laboratories, West Logan Cache, UTAH, and USA). Sections were deparaffinized with xylene and alcohol. After deparaffinization, endogenous peroxidase activity was blocked by treatment with $10 \%$ hydrogen peroxide for 10 minutes at room temperature. Sections were then subjected to high-temperature antigen retrieval by submerging in a boiling solution of $10 \mathrm{mM}$ citrate buffer ( $\mathrm{pH}$ 6.0) in a microwave oven 3 times with 5 minute intervals. After cooling at room temperature, sections were washed with $10 \mathrm{mM}$ phosphatebuffered saline (PBS, pH 7.5) and then incubated with optimal dilutions of primary antibodies overnight.

CD1a (clone O10), CD3 (clone RM-9107-R7), CD4 (clone 4B12), CD8 (clone C8/144B), CD25 (clone IL2R.1), CD14 (clone 14C02), and CD68 (clone KP1) were the primary antibodies used for immunohistochemical staining (NeoMarkers, Fremont, CA, USA).

Following incubation with optimal dilutions of primary antibodies, sections were washed with PBS for $3 \times 2$ minutes, and then incubated with biotinized secondary antibodies for 10 minutes. Subsequently, sections were again washed with PBS for $3 \times 2$ minutes, and incubated in streptavidin-peroxidase conjugate for 10 minutes. After three 2 minute washes in PBS, antibody-binding sites were demonstrated with soaking the sections in aminoethylcarbazole (AEC) horseradish peroxidase substrate for 10 minutes. Finally, sections were washed with PBS for 3 x 2 minutes, and then counterstained with Mayer's hematoxylin and mounted in aqueous mounting medium. Non-immune mouse serum was used as the negative control instead of primary antibodies.

\section{Evaluation of immune reactivity}

CD1 monoclonal antibodies were used to identify Langerhans cells; these cells are powerful antigen presenting cells (APCs) of the skin and mucosa. CD3 is a pan-T-cell specific monoclonal antibody, CD4 is present in $\mathrm{T}$ (helper/inducer) cells, CD8 is present in $\mathrm{T}$ (suppressor/cytotoxic) cells, $\mathrm{CD} 25$ is present in regulatory $\mathrm{T}$ cells and is an activation antigen, CD14 is a marker of the cells of myelomonocytic lineage (monocytes, macrophages, and Langerhans cells), and CD 68 is a histiocytic marker.

In order to determine the status of cell populations, a morphometric examination was performed blind to the clinical and morphologic characteristics. The full-thickness sections were evaluated by dividing into the following three layers: epithelium, superficial submucosa immediately underlying the epithelium, and deep submucosa lying immediately adjacent but distal to the glandular region. The number of cells in each layer was counted under a light microscope at 400X magnification (40X objective lens and 10X ocular lens). The area of each layer was measured using ImageJ software program (Version 1.38x; National Institutes of Health, Bethesda, MD, USA). The number of cells per $\mathrm{mm}^{2}$ was separately calculated for the epithelium, superficial sub mucosa, and deep sub mucosa.

\section{Statistical analysis}

Statistical analysis was conducted using SPSS for Windows (Version 16; SPSS Inc., Chicago, IL, USA). The number of cells per $\mathrm{mm}^{2}$ in the $\mathrm{AR}, \mathrm{IR}$, and control groups was compared using the Kruskal-Wallis non-parametric method. A p value $<0.05$ was considered statistically significant.

\section{Results}

Three groups are formed as 'allergic rhinitis', 'idiopathic rhinitis' and 'control' group and subjects are classified into one of these. Clinical and histochemical data of patients with idiopathic rhinitis, allergic rhinitis and control subjects are presented in table 1. 
Table 1: Clinical and histochemical data of patients with idiopathic rhinitis allergic rhinitis and control subjects

\begin{tabular}{|l|l|l|l|}
\hline & $\begin{array}{l}\text { Allergic rhinitis } \\
(\mathbf{n = 1 6})\end{array}$ & $\begin{array}{l}\text { Idiopathic rhinitis } \\
(\mathbf{n = 1 7})\end{array}$ & $\begin{array}{l}\text { C o } \mathbf{~ t ~} \text { o I } \\
(\mathbf{n}=\mathbf{2 5})\end{array}$ \\
\hline Gender (M/F); $\mathrm{n}$ & $6 / 10$ & $9 / 8$ & $15 / 10$ \\
\hline Age; years, min-max (mean) & $15-32(21)$ & $18-41(25)$ & $20-48(29)$ \\
\hline Duration of symptoms; years & $>2$ & $>2$ & - \\
\hline
\end{tabular}

Table 2: The number of leukocyte-lymphocyte marker-positive cells

\begin{tabular}{|c|c|c|c|c|}
\hline Marker & Group & $\begin{array}{l}\text { Epithelium (cell/ } \\
\mathrm{mm}^{2} \text { ), } \mathrm{n}\end{array}$ & $\begin{array}{l}\text { Superficial } \\
\text { submucosa (cell/ } \\
\text { mm²), n }^{2}\end{array}$ & $\begin{array}{l}\text { Deep } \\
\text { submucosa } \\
\left(\text { cell } / \mathrm{mm}^{2}\right), \mathrm{n}\end{array}$ \\
\hline \multirow{3}{*}{ CD1 } & CG & 21.12 & 3.20 & 1.68 \\
\hline & IR & 54.76 & 4.06 & 5.82 \\
\hline & AR & 51.19 & 5.88 & 4.19 \\
\hline \multirow{3}{*}{ CD3 } & CG & 558.52 & 682.76 & 559.08 \\
\hline & IR & 576.41 & 529.53 & 482.76 \\
\hline & AR & 542.94 & 478.38 & 446.69 \\
\hline \multirow{3}{*}{ CD4 } & CG & 366.64 & 426.92 & 388.00 \\
\hline & IR & 358.76 & 304.76 & 392.35 \\
\hline & AR & 295.56 & 279.63 & 274.75 \\
\hline \multirow{3}{*}{ CD8 } & CG & 333.56 & 274.08 & 231.52 \\
\hline & IR & 331.41 & 270.24 & 247.59 \\
\hline & AR & 422.69 & 396.13 & 359.88 \\
\hline \multirow{3}{*}{ CD25 } & CG & 9.96 & 6.96 & 4.76 \\
\hline & IR & 0.76 & 1.59 & 0.88 \\
\hline & AR & 0.31 & 1.75 & 1.88 \\
\hline \multirow{3}{*}{ CD14 } & CG & 296.52 & 278.16 & 255.32 \\
\hline & IR & 193.94 & 175.29 & 160.18 \\
\hline & AR & 222.31 & 183.25 & 159.25 \\
\hline \multirow{3}{*}{ CD68 } & CG & 111.96 & 93.36 & 78.04 \\
\hline & IR & 124.71 & 104.76 & 92.71 \\
\hline & AR & 116.81 & 103.13 & 83.19 \\
\hline
\end{tabular}

(CG: Control group, IR: Idiopathic rhinitis, AR: Allergic rhinitis)

The number of each inflammatory cell subgroups are counted in three layers and presented in table 2

Ciliated columnar epithelial cells, goblet cells, and pseudostratified prismatic epithelium were present in the epithelial layer. Cell-rich loose connective tissue was present in the superficial submucosal layer, whereas relatively cell-poor and partially collagenized connective tissue existed in the deep submucosal layer. All of the sections were thick enough to evaluate the three layers. The immunohistochemical staining method revealed red-stained cells on a blue base.

\section{T-lymphocytes}

These small, round-shaped cells were detected in all three of the layers. These cells were either scattered or aggregated. The number of CD3-, CD4-, CD8-, and CD25-positive cells per $\mathrm{mm}^{2}$ is shown in figure 1-4.

In epithelium and deep submucosa of patients with allergic and idiopathic rhinitis the numbers of CD3-positive cells were not significant. Only in sub mucosa there were significantly less CD3positive cells both in AR and IR ( $p<0.05)$. There was no difference between AR and IR $(p<0.05)$. There was no significant difference between the three groups in terms of the number of CD4-positive cells in the epithelium of the nasal mucosa, whereas a significant increase in the number of CD4-positive cells in the superficial submucosa was noted in patients with control group compared to the AR and IR groups $(\mathrm{p}<0.05)$. In all submucosal layers more CD8-positive cells has been detected in AR than control group and IR counterparts. There was no statistically meaningful difference between IR and control subjects. CD25-positive cells were significantly less in AR and IR group than control subjects. This was valid for all three mucosal layers.

\section{Langerhans cells}

These dendritic cells were mostly present in the epithelial layer, and rarely existed in the superficial or deep submucosa. The number of CD1-positive cells per $\mathrm{mm}^{2}$ is shown in figure 5 .

The number of CD1-positive cells in the epithelial and deep submucosal layer was significantly higher in the IR and AR groups as compared to the healthy control group $(\mathrm{p}<0.05)$.

CD1-positive cells, markers of APCs, were significantly increased within the deep submucosa of the AR and IR groups as compared to the control group. There was no significant difference between the patients with IR and AR regarding the number of CD1-positive cells per $\mathrm{mm}^{2}$

\section{Macrophage and monocytes}

Macrophage and monocytes were present in the epithelial, superficial, and deep submucosal layers. The macrophages and monocytes were detected in the superficial and deep submucosa. The numbers of CD14- and CD68-positive cells per $\mathrm{mm}^{2}$ are presented in figures 6 and figure 7.

More CD14-positive cells, markers of myelomonocytic cells, were present in all three layers of the AR and IR groups as compared to the control group $(\mathrm{p}<0.05)$. The presence of large numbers of CD14positive cells, which play a role in the processing and presentation of nasal antigens, indicated an inflammatory pathology. There was no significant difference between the groups regarding the number of CD68-positive cells per $\mathrm{mm}^{2}$.

\section{Discussion}

When rhinitis is classified according to etiology, patients with non-allergic, non-infectious rhinitis are considered non-atopic because there is no evidence of an IgE-dependent systemic response. The term 'idiopathic rhinitis' defines a subgroup of patients with unknown etiology among patients with non-allergic, non-infective rhinitis. Patients with IR account for approximately $60 \%$ of patients with non-allergic rhinitis and approximately $36 \%$ of all rhinitis sufferers. A number of terms have been recently suggested to define this group of patients. The term "vasomotor rhinitis" is a used one; however, this term has been suggested to be reserved for rhinitis in which a neurogenic pathophysiology is involved [4]. Similarly, the differentiation of patients with non-allergic rhinitis with eosinophilia syndrome (NARES) from IR patients is controversial since the reason for eosinophil accumulation in the nasal mucosa of these patients and the underlying mechanisms are not known.

Neural dysregulation of the nasal mucosa has been suggested as an underlying pathophysiologic mechanism in patients with IR. This opinion supports the notion that autonomic dysfunction or hyperactive non-adrenergic non-cholinergic peptidergic neural system causes symptoms in IR [10-13]. Although the studies are aimed at elucidating the mechanism of autonomic dysfunction or change in the number of unmyelinated C-fibers, it is incredibly difficult to prove this. On the other hand, there are studies explaining the clinical efficacy of repeated intranasal capsaicin application with neurogenic mechanisms. Fokkens et al. and Wolf et al. suggested that autonomic nervous system disorders that result in hypoactivity of the sympathetic nervous system or hyperactivity of the peptidergic system are responsible for the pathophysiology in patients with IR $[14,15]$.

There are many studies suggesting a local allergic response in patients with IR, despite the negative results of systemic tests for atopy. Various methods have been used in those studies; specific IgE or inflammatory mediators have been investigated in nasal secretions, the symptoms of rhinitis have been evaluated following nasal allergen provocation, immunohistochemical examinations of the nasal mucosa have been performed, and specific allergen-binding properties have been evaluated in non-atopic patients.

In the current study, the inflammatory cells that were newly formed or increased in number during the allergic response were investigated in biopsy specimens obtained from the patients with perennial AR and IR and compared with normal mucosal cells. 


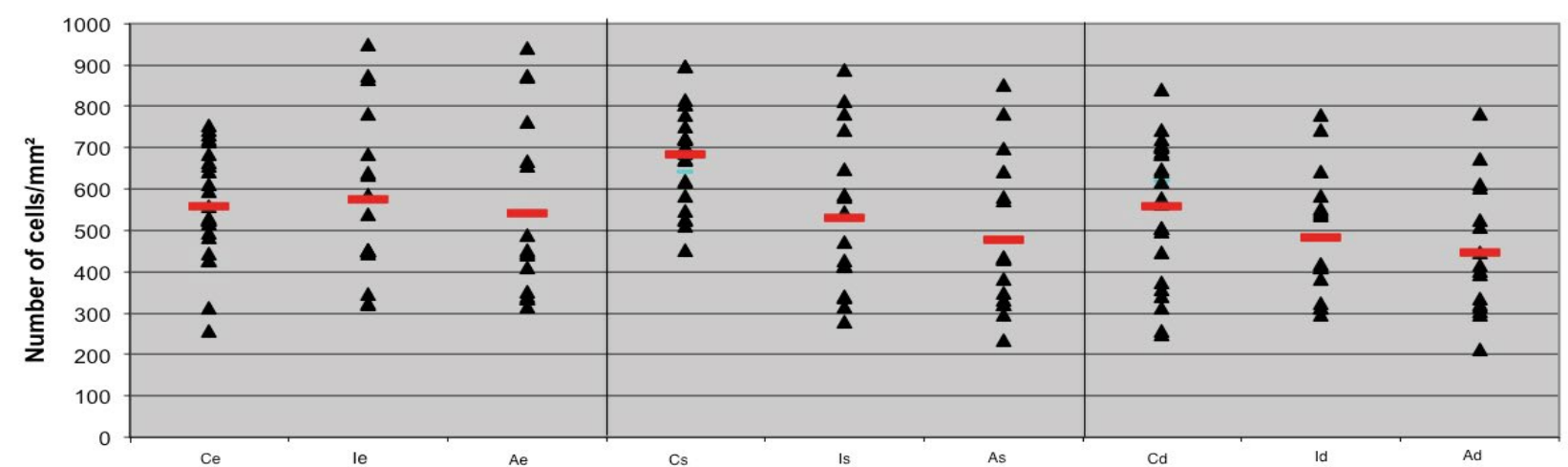

EPITHELIUM

SUPERFICIAL SUBMUCOSA

DEEP SUBMUCOSA

CD4

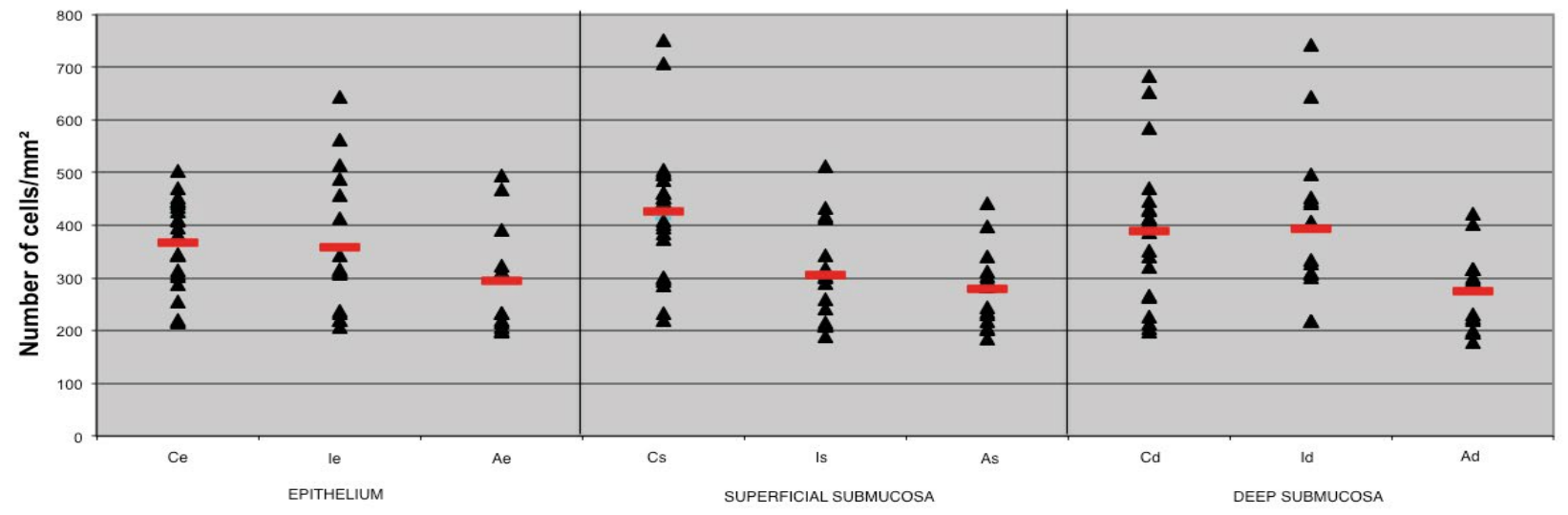

CD8

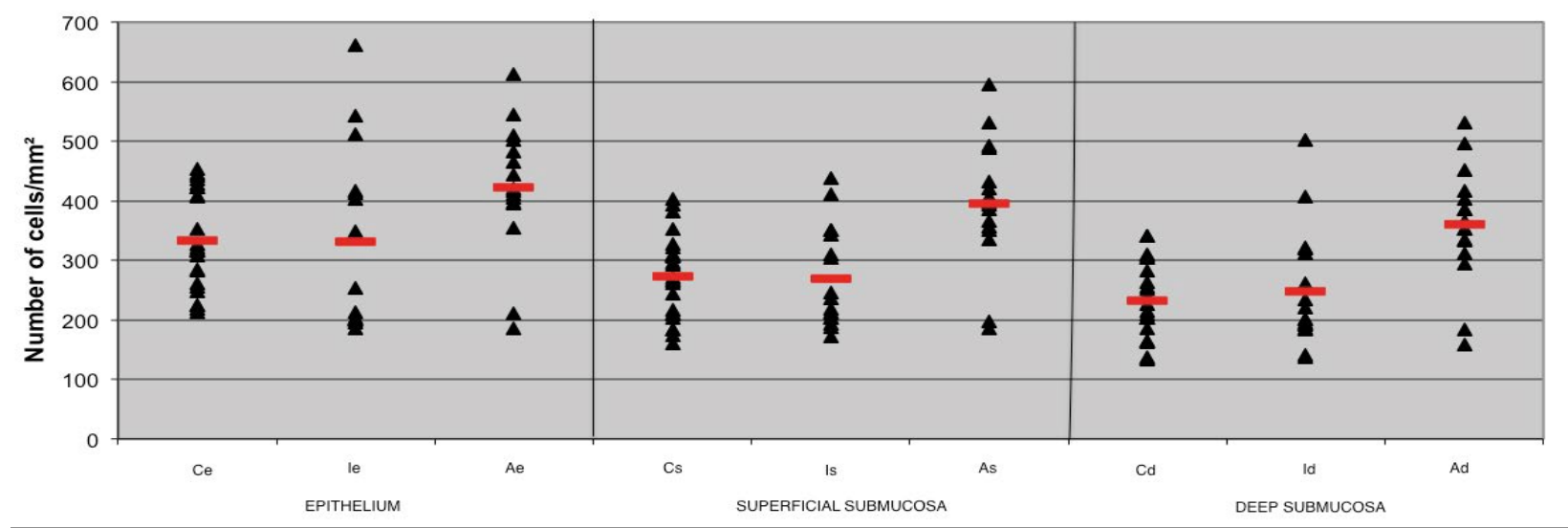

CD25

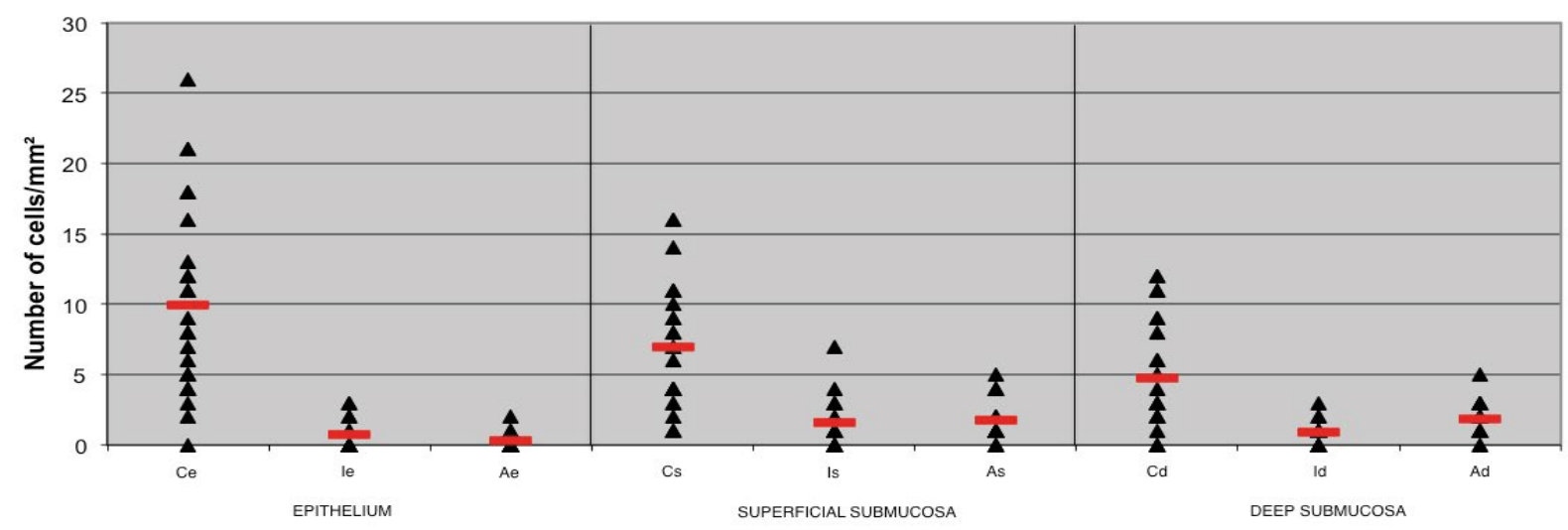

Figures 1-4: The number of CD3-, CD4-, CD8-, and CD25- positive cells per mm². C: the control group, I: the idiopathic rhinitis group, A: the allergic rhinitis group, e: epithelium, s: superficial submucosa, d: deep submucosa. 


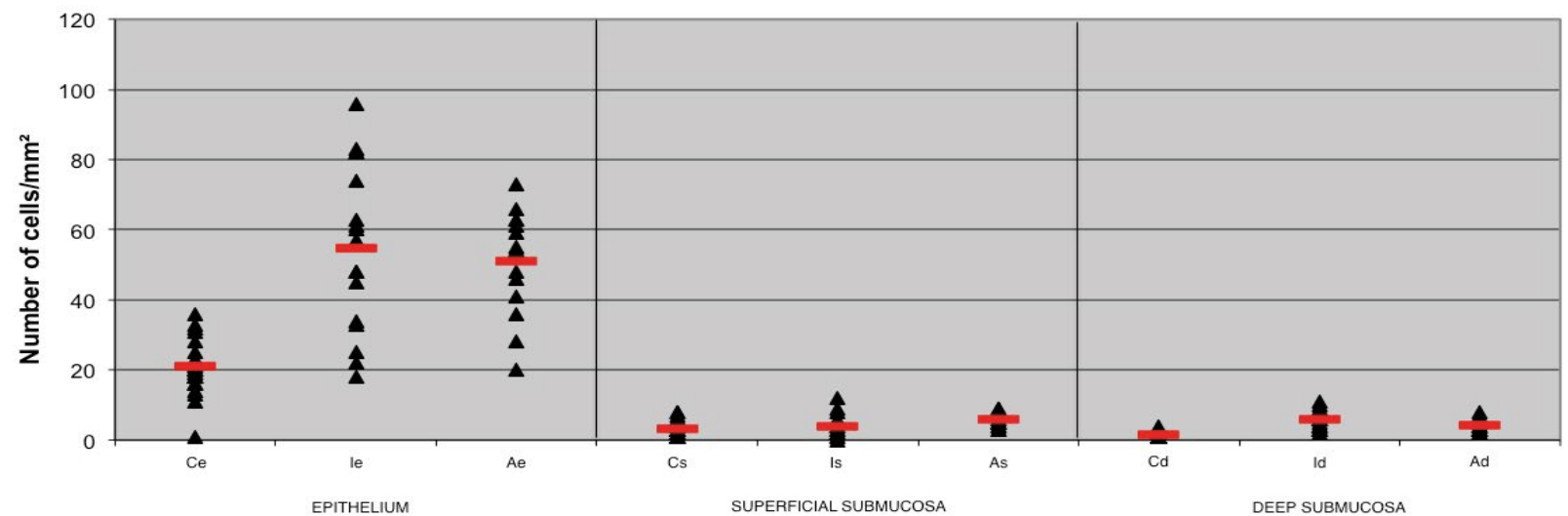

Figure 5: The number of CD1-positive cells per $\mathrm{mm}^{2}$. C: the control group, I: the idiopathic rhinitis group, A: the allergic rhinitis group, e: epithelium, s: superficial submucosa, d: deep submucosa.

CD14
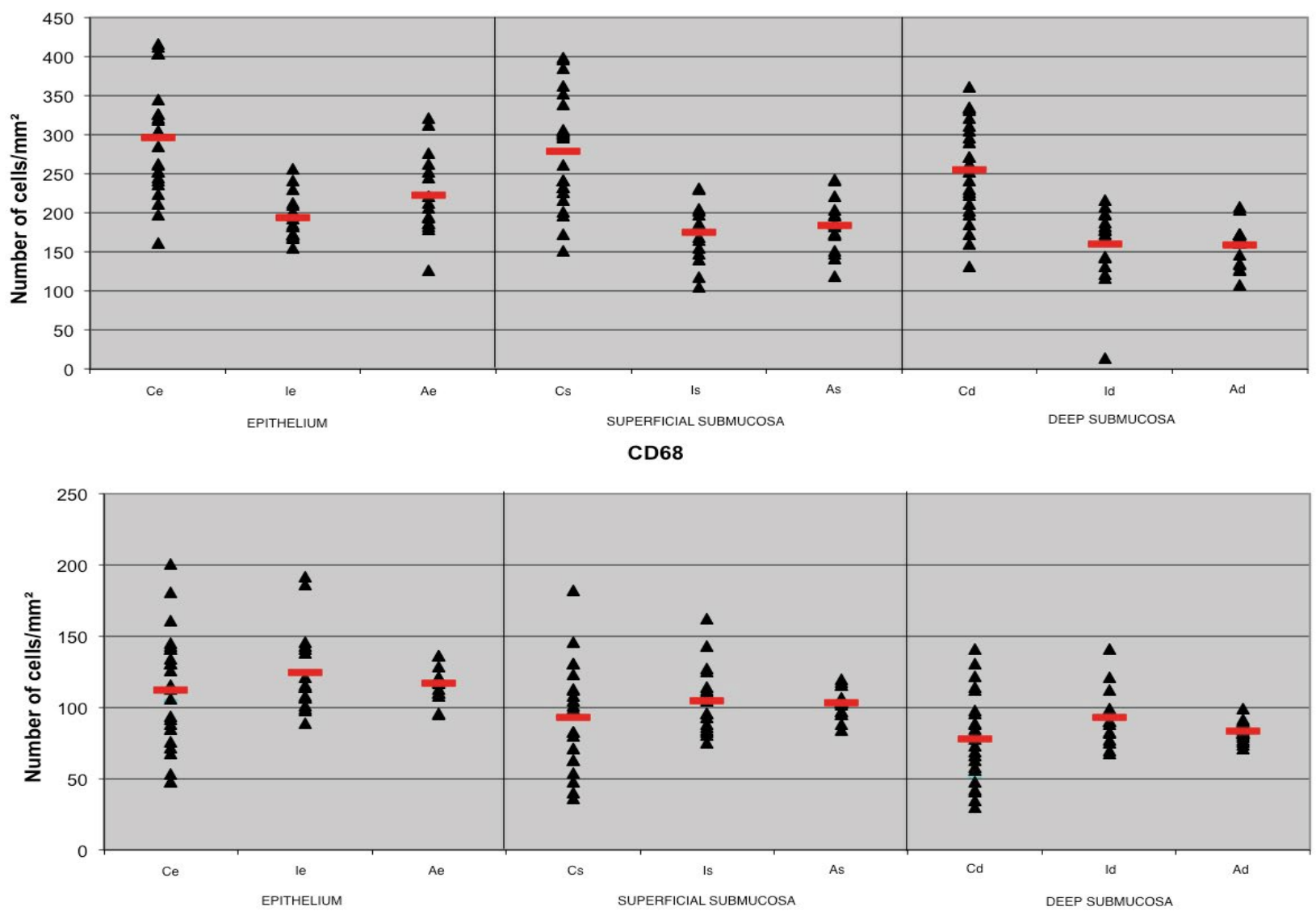

Figure 6 and 7: The number of CD14- and CD68-positive cells per $\mathrm{mm}^{2}$. C: the control group, I: the idiopathic rhinitis group, A: the allergic rhinitis group,

e: epithelium, s: superficial submucosa, d: deep submucosa.

Moreover, the inflammatory patterns of both forms of rhinitis were studied.

CD1, which is used as a marker of APCs in the nasal mucosa, has been used to identify Langerhans cells. It has been shown that APCs decrease the Th1/Th2 balance by reducing the production of IL-2, which activates Th1 cells, and increase the immune response, in which Th2 cells are involved [16,17]. In this current study, APCs were higher in the epithelium of AR patients compared to the control group. Moreover, APCs were significantly increased also in the nasal mucosal epithelium of IR patients as compared to the control group $(\mathrm{p}<0.05)$. In patients with IR, APCs were significantly increased both within the epithelium and deep submucosa. Increased number of
APC's with respect to control group has been detected in epithelial and deep submucosal layer of mucosa of IR and AR patients. Numbers of APC's were also high in superficial submucosal layer of mucosa of AR and IR patients when compared to control subjects but the difference was not statistically significant. This is likely to determine the pattern and intensity of the inflammation. In nasal mucosa of patients with idiopathic rhinitis APC's seem to distribute in similar pattern with allergic counterparts.

CD3 is a specific marker present in all T cells. In the current study, more CD3-positive cells were noted in the nasal epithelium compared to the superficial and deep submucosal layers of the IR and allergic rhinitis groups. On the other hand, more CD3-positive 
cells were present in the superficial submucosal layer compared to the epithelial and deep submucosal layers of the control group. In $\mathrm{AR}$ and IR patients contribution of CD3-positive cells seems to be similar as majority of cells accumulated in epithelial layer. Numbers of CD3-positive cells in AR and IR were less than control subjects in superficial submucosal layer of mucosa $\left(478,38\right.$ cells $/ \mathrm{mm}^{2}$ and 529,53 cells $/ \mathrm{mm}^{2}$ and 682,76 cells $/ \mathrm{mm}^{2}$ respectively) this difference was the only statistically significant one in CD3 counts $(\mathrm{p}<0.05)$. But there seem to be a tendency to accumulate in epithelial layer in epithelial layer in AR and IR patients. This finding gave rise to thought that CD3-positive cells in IR and AR groups tend to be close to allergens.

CD4-positive Th cells participate in the allergic response by secreting cytokines. CD4-positive Th cells account for $<5 \%$ of CD3positive $\mathrm{T}$ cells in peripheral circulation, and they increase in number in the nasal mucosa during the allergic response in patients with perennial AR and seasonal AR [18]. The number of CD4-postive cells has generally been shown to be higher than the number of CD8positive cells in patients with AR and in those with IR. In the current study, there was no significant difference between the three groups in terms of the number of CD4-positive cells in the epithelium of the nasal mucosa, whereas a significant increase in the number of CD4positive cells in the superficial submucosa was noted in patients with control group compared to the AR and IR groups (426, 92 cells $/ \mathrm{mm}^{2}$ and 279,63 cells $/ \mathrm{mm}^{2}$ and 304,76 cells $/ \mathrm{mm}^{2}$ respectively). Moreover, more CD4-positive cells were observed in the deep submucosa of patients with control and IR group compared to the AR group. No significant difference was found between the patients with control and IR group regarding the number of CD4-positive cells in the superficial submucosa. In contrast to the clinical provocation studies in which a significant increase in the number of CD4-positive cells in the epithelium had been determined [19], the lack of such increase in patients with AR in the current study could be attributed to the fact that the patients had not been exposed to allergens prior to the biopsy. Despite the number of CD4-positive cells is low in AR group, cells tend to accumulate coming close to surface of mucosa. This finding is same as passing from superficial submucosa to epithelium in IR patients. In the current study, the highest number of CD8-positive cells was noted in the nasal mucosa of the patients with AR. The most remarkable difference in inflammatory character of AR patients was abundance of CD8-positive cells and the number of these cells was significantly higher in all three layers of mucosa. These cells are defined as cytotoxic T cells and generally participate in the recognition of the antigens associated with MHC class 1 protein. Although mature Th cells express the surface protein CD4, and known as CD4-positive Th cells; it has been demonstrated that cytotoxic T cells are also able to express CD4 [20]. The number of CD8-positive cells of allergic patients is higher than those of idiopathic rhinitis and the difference is significant. This finding seems to be remarkable and distinctive. Moreover there was no difference between patients with idiopathic rhinitis and control subjects. The number of CD25-positive cells was found lower in the epithelium, superficial and deep submucosa of patients with AR and IR as compared to the control group. CD25positive cells have regulatory functions such as to suppress induction of effector lymphocytes. Both in AR and IR group lack of these cells might contribute to induction of cellular immune response. There was no difference $(\mathrm{p}<0.05)$ between the IR and AR groups regarding the number of CD25-positive cells in all three mucosal layers.

In the current study, the number of CD14-positive cells in all three layers of the nasal mucosa was found lower in the IR and AR groups as compared to the control group ( $p<0.05)$. CD14-positive cells represent monocytes, macrophages, granulocytes and B-lymphocytes. As a membrane glycoprotein it acts as an inducer of interleukin 12 (IL12) which takes role in maturation of naïve $T$ cells into $T h-1$ cells and down regulation of Th-2 cells. This alteration shifts Th1/Th2 balance against Th2 and eventually reduces production of IgE. No difference was noted between the IR and AR groups regarding the number of CD14-positive cells $(p<0.05)$. Further studies are warranted to understand the pathophysiologic importance of the above mentioned findings. Although the importance of the decrease in the density of myelomonocytic cells remains unknown, it is remarkable that the patients with AR and IR share a similar inflammatory pattern.

Although the number CD68-positive cells, including monocytes and tissue macrophages, was slightly increased in the AR and IR groups compared to the control group, the difference did not reach statistical significance. Despite a similar inflammatory pattern is suggested for CD68, further studies are needed on this issue.

In recent years, various studies have reported that patients with IR and AR show a very similar leukocyte-lymphocyte inflammatory pattern following nasal provocation. Rondón et al. [21] and Carmen et al. [22] suggested defining these patients, in whom similar responses have been obtained with nasal provocation tests, as having "local AR." The inflammatory pattern in nasal secretions of patients with seasonal symptoms and a negative SPT, following nasal provocation has been investigated and found to be similar to those of patients with AR. In the current study, there was no periodicity in the symptoms of patients with IR, and samples for histological examinations from AR patients were obtained during symptomatic periods. These findings reveal that patients with IR give a tissue response against the allergen in some way; however, the inflammation is limited to the nasal mucosa in these patients and a response cannot be obtained in the diagnostic skin tests.

\section{Acknowledgements}

The authors would like to thank Mr. Ali Çağrı Bozdoğangil, for assisting with preparation of the manuscript, for providing statistical analysis and invaluable logistical support. Our study is not sponsored by any organization or firm.

\section{Authorship Contribution}

Specific contributions of all authors are as fallows. Şenol Çomoğlu took role in collecting data, analysing of the data for the work, drafting the work and final approval. Ece Comoğlu contributed to collecting data, drafting the work and final approval. M. Nesil Keles has designed the study, drafted the work and contributed to final approval. Kemal Değer contributed to design of the study, drafting the work and final approval.

\section{Conflict Of Interest}

There is no conflict of interest or financial affiliance.

\section{References}

1. Carney AS, Jones NS (1996) Idiopathic rhinitis: idiopathic or not? Clin Otolaryngol 21:198-202

2. Carney AS, Powe DG, Huskisson RS, Jones NS (2002) A typical nasal challenges in patients with idiopathic rhinitis: more evidence for the existence of allergy in the absence of atopy? Clin Exp Allergy 32: 1436-1440.

3. Powe DG, Huskisson RS, Carney AS, Jenkins D, Jones NS (2001) Evidence for an inflammatory pathophysiology in idiopathic rhinitis. Clin Exp Allergy 31: 864-872.

4. Powe DG, Jones NS (2006) Local mucosal immunoglobulin E production does allergy exist in non-allergic rhinitis? Clin Exp Allergy 36: 1367-1372.

5. Durham SR, Smurthwaite L, Gould HJ (2000) Local IgE production. Am J Rhinol 14: 305-307.

6. Powe DG, Huskisson RS, Carney AS, Jenkins D, Jones NS (2001) Evidence for an inflammatory pathophysiology in idiopathic rhinitis. Clin Exp Allergy 31: $864-872$

7. Powe DG, Jagger C, Kleinjan A, Carney AS, Jenkins D, et al. (2003) 'Entopy' localized mucosal allergic disease in the absence of systemic responses for atopy. Clin Exp Allergy 33: 1374-1379.

8. Rondón C, Fernández J, López S, Campo P, Doña I, et al. (2009) Nasal inflammatory mediators and specific IgE production after nasal challenge with grass pollen in local allergic rhinitis. J Allergy Clin Immunol 124: 1005-1011.

9. Powe DG, Groot Kormelink T, Sisson M, Blokhuis BJ, Kramer MF, et al. (2010) Evidence for the involvement of free light chain immunoglobulins in allergic and nonallergic rhinitis. J Allergy Clin Immunol 125: 139-145.

10. Baraniuk JN, Kaliner MA (1990) Neuropeptides and nasal secretion. J Allergy Clin Immunol 86: 620-627.

11. Fang SY, Shen CL (1998) Neuropeptide innervation and neuroendocrine cells in allergic rhinitis and chronic hypertrophic rhinitis. Clin Exp Allergy 28: 228-232.

12. Lacroix JS, Kurt AM, Pochon N, Bretton C, Lundberg JM, et al. (1995) Neutra endopeptidase activity and concentration of sensory neuropeptide in the human nasal mucosa. Eur Arch Otorhinolaryngol 252: 465-468. 
13. Wolf $\mathrm{G}$ (1988) New aspects in the pathogenesis and therapy of hyperreflexive rhinopathy. Laryngol Rhinol Otol (Stuttg) 67: 438-445.

14. Van Rijswijk JB, Boeke EL, Keizer JM, Mulder PG, Blom HM, et al. (2003) Intranasal capsaicin reduces nasal hyperreactivity in idiopathic rhinitis: a double-blind randomised application regimen study. Allergy 58: 754-761.

15. Wolf $\mathrm{G}$ (1988) New aspects in the pathogenesis and therapy of hyperreflexive rhinopathy. Laryngol Rhinol Otol (Stuttg) 67: 438-445.

16. Reider N, Reider D, Ebner S, Holzmann S, Herold M, et al. (2002) Dendritic cells contribute to the development of atopy by an insufficiency in IL-12 production. J Allergy Clin Immunol 109: 89-95.

17. Ghaemmaghami AM, Gough L, Sewell HF, Shakib F (2002) The proteolytic activity of the major dust mite allergen Der p 1 conditions dendritic cells to produce less interleukin-12: allergen-induced Th2 bias determined at the dendritic cell level. Clin Exp Allergy 32: 1468-1475.
18. Calderón MA, Lozewicz S, Prior A, Jordan S, Trigg CJ, et al. (1994) Lymphocyte infiltration and thickness of the nasal mucous membrane in perennial and seasonal allergic rhinitis. J Allergy Clin Immunol 93: 635-643.

19. Ghaemmaghami AM, Gough L, Sewell HF, Shakib F (2002) The proteolytic activity of the major dust mite allergen Der $p 1$ conditions dendritic cells to produce less interleukin-12: allergen-induced Th2 bias determined at the dendritic cell level. Clin Exp Allergy 32: 1468-1475.

20. Amin K, Rinne J, Haahtela T, Simola M, Peterson CG, et al. (2001) Inflammatory cell and epithelial characteristics of perennial allergic and nonallergic rhinitis with a symptom history of 1 to 3 years' duration. J Allergy Clin Immunol 107: 249-257.

21. Rondón C, Fernandez J, Canto G, Blanca M (2010) Local allergic rhinitis: concept, clinical manifestations, and diagnostic approach. J Investig Allergo Clin Immunol 20: 364-371.

22. Rondón C, Canto G, Blanca M (2010) Local allergic rhinitis: a new entity characterization and further studies. Curr Opin Allergy Clin Immunol 10: 1-7. 